\title{
Ionic liquids for the control of the morphology in poly(vinylidene fluoride-co-hexafluoropropylene) membranes
}

\author{
Paula GSaiz $^{1}$, Ana Catarina Lopes ${ }^{1 *}$, Simone Eizagirre ${ }^{1}$, Roberto Fernández de Luis ${ }^{1}$, María Isabel \\ Arriortua $^{1,2}$ \\ ${ }^{1}$ BCMaterials (Basque Center for Materials, Applications \& Nanostructures), Bldg. Martina Casiano, 3rd. Floor, \\ UPV/EHU Science Park, Barrio Sarriena S/N, Leioa, 48940, Spain. \\ ${ }^{2}$ Mineralogy and Petrology Department, University of the Basque Country (UPV/EHU), Barrio Sarriena S/N, \\ Leioa, 48940, Spain.
}

\begin{abstract}
:
The development of polymer membranes with tailored micro-morphology and wettability is a demand in the areas of filtration, sensors, and tissue engineering, among others. The thermoplastic copolymer poly(vinylidene fluoride-cohexafluoropropylene) (PVDF-HFP), is one of the most widely used polymers for these applications due to its good mechanical and thermal properties, biocompatibility and low density. Although the control of the PVDF-HFP morphology is a complicated task, the introduction of ionic liquids (ILs) in the PVDF-HFP matrix opens news perspectives in this area. This work consists on a systematic study of three different protic ionic liquids ([dema][TfO], [MIm][NTf2] and [MIm][Cl]) in the control of PVDF-HFP membranes properties. Different preparation conditions are also analysed. These results demonstrate how several parameters such as morphology, water absorption capacity and mechanical properties vary depending on the production methodology employed and on the choice of incorporated IL. Pores of different sizes, spherulites, and compact structures have been obtained, as well hydrophilic and highly hydrophobic structures. These results show that ILs play a key role in the optimization of polymer properties, and given the huge number of ILs available, they open up new possibilities for the development of polymer membranes suitable for applications where specific morphologies are desirable.
\end{abstract}

\section{Keywords:}

Poly(vinylidene fluoride-co hexafluoropropylene)- PVDF-HFP; Modelling morphology; Ionic liquids; Membranes; Mechanical properties; Hydrophilic properties.

\section{Introduction}

Nowadays, the development of polymer membranes with tailored micro-morphology, mechanical properties or water absorption capacity is a demand in the areas of filtration, electronics, sensors, tissue engineering or energy, among others [13]. Morphology characteristics significantly determine the final mechanical, thermal, optical and electronic properties of membranes and for this reason many efforts have been devoted to understanding how to control and tailor membrane morphology, [4-5], as this could open new possibilities in the previously cited applications [6-7].

Some of the most commonly used polymers in membrane development are polyvinylidene fluoride (PVDF) and its copolymers. They are semicrystalline thermoplastic fluoropolymers with good physical and chemical properties, including good chemical and thermal stability, high polarity, low density, hydrophobic nature and biocompatibility [8]. Particularly, this family of polymers stands out for having the highest dielectric constant and the highest piezoelectric response among all the polymers. PVDF and its co-polymers are polymorphous polymers that can exhibit five crystalline phases, including two piezoelectric ( $\gamma$ and $\beta$-phases), which can be obtained by the control of the production method. The piezoelectric phases are 
widely applied in the area of sensors and actuators [9] as well as emerging applications such as the development piezoelectric scaffolds for tissue regeneration in biomedical applications [10].

In the particular case of the copolymer poly(vinylidene fluoride-co-hexafluoropropylene) (PVDF-HFP), the inclusion of hexafluoropropylene (HFP) in the structure results in a more hydrophobic film and a lower melting point due to a decrease in the degree of crystallinity with respect to the homopolymer [11]. These characteristics make PVDF-HFP particularly interesting in the area of battery separator membranes [12] and membrane distillation [13], among others. The control of PVDF membrane morphology is commonly a complicated task mostly restricted to the use of solvent evaporation (SE), thermally induced phase separation (TIPS), diffusion induced phase separation (DIPS) and non-solvent-induced phase separation (NIPS) techniques, where variable processing parameters are mostly limited to evaporation temperature, type of solvent or reaction time [14-17]. Furthermore, these techniques mostly result in very non-uniform porosities through the thickness of membranes as the contact with the atmosphere or non-solvent takes place just at membrane surface [8].

The use of a filler that can induce a uniform microstructure from all over the membrane could provide a more efficient morphology control method. Fillers such as clays [18] or metal nanoparticles [19] are usually added in order to introduce new or desired properties to the polymers (higher resistance, conductivity, larger dielectric response...) [20]. One of the most promising materials for this purpose are ionic liquids (ILs). These materials, commonly defined as molten salts with a melting point lower than $100^{\circ} \mathrm{C}$, are receiving large attention on a wide variety of applications including solvents [21], energy [22], gas separation [23] or even in biochemical processes [24], due to their high thermal and electrochemical stability, low vapor pressure, high ionic conductivity and non-flammability. ILs have already shown the capacity to affect the morphology and size of nanoparticles when used during synthesis [25]-[27] as well as the morphology of some polymer membranes [28]. However, their capacity to induce a specific morphology has only been studied as a parallel effect [29], [30]. There has not, so far, been performed a systematic study about this important ability of ILs. In this work, a comprehensive and systematic study of the influence of ILs on the morphology, hydrophobicity, and thermal and mechanical properties of PVDF-HFP is presented through the production of PVDF-HFP@IL composite membranes with three ILs (diethylmethylmethylammonium trifluoromethanesulfonate, 1-methylimidazolium bis(trifluoromethylsulfonyl)imide and 1-methylimidazolium chloride) and varying methodologies (time and temperature of solvent evaporation).

The results bring out the ability of ILs to tailor the morphology and associated properties of polymer membranes. Taking into account that there exist more than $10^{3}$ different ILs [31], this work shows the possibility to tailor the most suitable combination of ILs to meet the desired properties of PVDF-HFP membranes for each specific applications.

\section{Experimental}

\subsection{Materials}

The ILs diethylmethylammonium trifluoromethanesulfonate ([dema][TfO]), 1-methylimidazolium bis(trifluoromethylsulfonyl)imide ([MIm] $\left[\mathrm{NTf}_{2}\right]$ ) and 1-methylimidazolium chloride ([MIm] [Cl]) were obtained from Iolitec (Germany) with stated purity of 98\%. Poly(vinylidene fluoride-co-hexafluoropropylene) (PVDF-HFP, SOLEF® 21216/1001 from Solvay (Belgium)) was used as polymer matrix and N,N-dimethylformamide (DMF) (99.8\%) (supplied by Sigma Aldrich) as solvent. The structures of the ionic liquids used are shown in the Figure 1. 
a)<smiles></smiles>

$\mathrm{Cl}^{-}$

b)<smiles>CC[NH+](C)CC</smiles>

c)

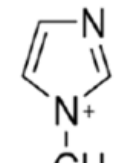<smiles>O=S(=O)([N-]S(=O)(=O)C(F)(F)F)C(F)(F)F</smiles>

$\mathrm{CH}_{3}$

Fig. 1 - Structure of: (a) [MIm][Cl], (b) [dema][TfO] and (c) [MIm] $\left.\mathrm{NTf}_{2}\right]$.

\subsection{Membrane preparation}

The membranes were prepared through solvent casting and subsequent evaporation of the solvent at different temperatures. $6 \mathrm{~mL}$ of solvent per gram of polymer were used, corresponding to a polymer:solvent ratio of 15:85 in weight. $60 \%$ wt of $[\mathrm{MIm}]\left[\mathrm{NTf}_{2}\right]$ was used to prepare the composite membranes. Equivalent molar quantities $\left(4.132 \cdot 10^{-3} \mathrm{moles} / \mathrm{gram}\right)$ of the other two ILs were used. The solvent, polymer and selected IL were mixed in the desired ratios and placed on a magnetic stirring plate at room temperature until the complete dissolution of the polymer was achieved. Then, the homogeneous solution was spread onto a glass substrate and the solvent evaporated either at room temperature or placed in the oven at a specific temperature for different periods of time $\left(80^{\circ} \mathrm{C}\right.$ for 20 minutes and in the oven at $100{ }^{\circ} \mathrm{C}$ for 1 hour) (Figure 2 ).
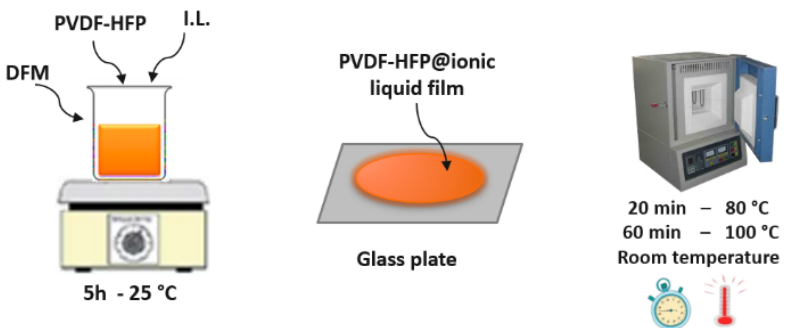

Fig. 2 - Schematic representation of the preparation method of PVDF-HFP@IL membranes.

The effect of different parameters on the properties of the samples was analyzed. In addition, the samples dried at $80{ }^{\circ} \mathrm{C}$ for 20 minutes were immersed in water for 48 hours to the complete removal of the IL and the resultant membranes were also subsequently characterized. The parameters of all the prepared samples are presented in Table 1. 
Table 1. Adjusted parameters on the samples preparation.

\begin{tabular}{|c|c|c|}
\hline Treatment & I.L. & Sample \\
\hline \multirow{4}{*}{ Room temperature } & - & PVDF-HFP_RT \\
\hline & {$[\mathrm{MIm}][\mathrm{Cl}]$} & PVDF-HFP@[MIm][Cl]_RT \\
\hline & [dema][TfO] & PVDF-HFP@[dema][Tfo]_RT \\
\hline & {$[\mathrm{MIm}][\mathrm{NTf} 2]$} & PVDF-HFP@[MIm][NTf $\left.f_{2}\right] \_R T$ \\
\hline \multirow{4}{*}{$80^{\circ} \mathrm{C}, 20 \mathrm{~min}$} & - & PVDF-HFP_80 $80^{\circ} \mathrm{C}$ \\
\hline & {$[\mathrm{MIm}][\mathrm{Cl}]$} & PVDF-HFP@[MIm][Cl]_80 $0^{\circ} \mathrm{C}$ \\
\hline & {$[$ dema $][T f O]$} & PVDF-HFP@[dema][TfO]_80 $8{ }^{\circ} \mathrm{C}$ \\
\hline & {$[\mathrm{MIm}][\mathrm{NTf} 2]$} & PVDF-HFP@[MIm][NTf $\left.{ }_{2}\right] \_80^{\circ} \mathrm{C}$ \\
\hline \multirow{4}{*}{$100^{\circ} \mathrm{C}, 60 \mathrm{~min}$} & - & PVDF-HFP_ $100^{\circ} \mathrm{C}$ \\
\hline & {$[\mathrm{MIm}][\mathrm{Cl}]$} & PVDF-HFP@[MIm][Cl]_100을 \\
\hline & [dema][TfO] & PVDF-HFP@[dema][TfO]_100 $10{ }^{\circ} \mathrm{C}$ \\
\hline & {$[\mathrm{MIm}][\mathrm{NTf} 2]$} & PVDF-HFP@[MIm] $\left.] \mathrm{NTf}_{2}\right] \_100^{\circ} \mathrm{C}$ \\
\hline
\end{tabular}

\subsection{Samples characterization}

In order to study the properties of the synthesized samples different measurements were carried out. The surface and transversal morphologies of the membranes were obtained using a Scanning Electron Microscope (SEM) (JEOL JSM-7000F) with an accelerating voltage of $5 \mathrm{kV}$. Prior to analysis, samples were coated with a gold layer of $15 \mathrm{~nm}$ using a sputter coating method. Contact angle measurements of the surface of membranes were recorded using the Neurtek OCA15EC Dataphysics instrument and deionized water drops of $2 \mu \mathrm{L}$. The presented average value of contact angle was calculated from five different measurements performed in each membrane. The mechanical properties of the membranes were analyzed by the trace of a stress-strain curve using a traction machine (Shimadzu AGS-500NJ) in the tensile mode. Samples with lengths and widths of $10 \mathrm{~mm}$ were stretched at a rate of $1 \mathrm{~mm} / \mathrm{min}$. The thermal properties of the membranes were studied by through thermogravimetric analysis (TGA), with the Netzsch STA 449F3 instrument. Small pieces of about 6 mg were cut, placed into aluminum oxide pans, and heated from 25 to $800^{\circ} \mathrm{C}$ at a heating rate of $5^{\circ} \mathrm{C} / \mathrm{min}$ under synthetic atmosphere. Finally, Fourier Transform Infrared Spectroscopy in Attenuated Total Reflectance mode (FTIR-ATR) measurements were carried out in order to determine the crystalline phase content of the composite membranes. The spectra were taken in the 600 and 4000 $\mathrm{cm}^{-1}$ range with a Jasco FT/IR-6100 spectrometer in ATR mode.

\section{Results and discussion}

\subsection{Morphology and hydrophobicity of the films}

Representative cross-sectional SEM images of the membranes as well as images of static contact angle measurements are shown in the Figure 3. The results reveal the existence of different morphologies and varying hydrophobic properties depending on the processing conditions and on the choice of IL. 


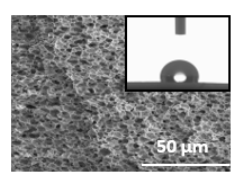

A1

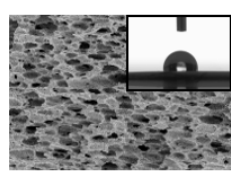

A2

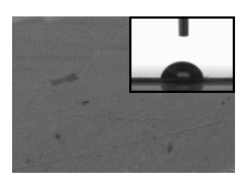

A3

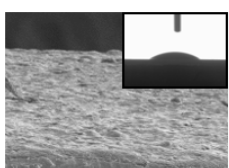

A4

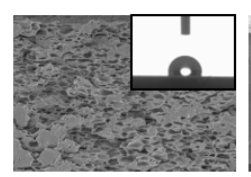

B1

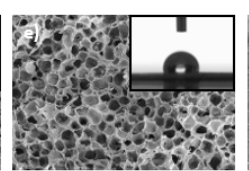

B2

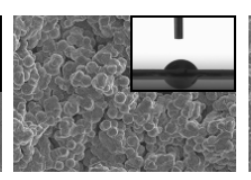

B3

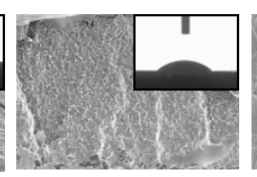

B4

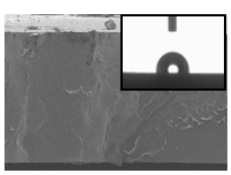

C1
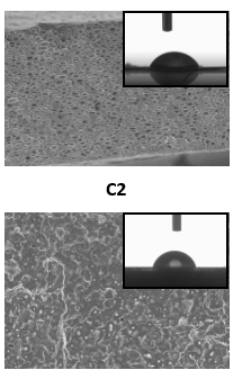

C3

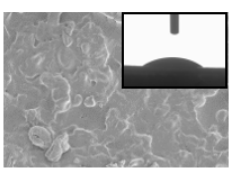

C4

Fig. 3 - Cross-sectional SEM and contact angle images for the different samples. Solvent evaporation temperature of A, B and $\mathrm{C}$ are $\mathrm{RT}, 80^{\circ} \mathrm{C}$ and $100^{\circ} \mathrm{C}$ respectively. $1,2,3$ and 4 represent the IL used for the preparation of the membrane (none, $[\mathrm{MIm}][\mathrm{Cl}]$, [dema][TfO] and $[\mathrm{MIm}]\left[\mathrm{NTf}_{2}\right]$ respectively).

As can be seen, temperature, and time of solvent evaporation play an important role on the morphology obtained. In the neat PVDF-HFP, the pore is formed by a phase separation process between the solvent and the polymer [32], such that the solvent evaporation rate plays a key role in the morphology of the obtained membrane. At higher temperatures, the DMF evaporation occurs quickly, resulting in membranes that are either non-porous or only have small pores. When the solvent evaporates slowly the process of phase separation is completed, resulting in a larger pore size membranes, as is effectively observed in the neat PVDF-HFP samples evaporated at room temperature, In these conditions, uniform membranes with a high porosity structure (Figure 3-A1) are achieved. However, as the evaporation temperature rises, some non-porous parts arise (Figure 3-B1) or a compact (non-porous) structure appears (Figure 3-C1).

A similar behavior is observed in the PVDF-HFP@[MIm] [Cl] composites where the pore size decreases as the temperature and time of solvent evaporation increase. Particularly, we observe that the sample dried at room temperature presents a medium pore size of $6 \pm 3 \mu \mathrm{m}$, which decreases to values of $5.0 \pm 1.3 \mu \mathrm{m}$ when the sample is dried at $80^{\circ} \mathrm{C}$. In the sample dried at $100{ }^{\circ} \mathrm{C}$, is evident an even more expressive reduction of the porous size to $2.4 \pm 0.9 \mu \mathrm{m}$.

Nonetheless, this behavior was not observed for composites with all the ILs. Contrary to the membranes of PVDF-HFP and PVDF-HFP@[MIm][Cl] which show a high degree of porosity, the membranes prepared with $[\mathrm{MIm}]\left[\mathrm{NTf} \mathrm{f}_{2}\right]$ and [dema][TfO] present a different structure. In the PVDF-HFP@[dema][TfO] composite, no pores are observed in SEM image when the sample is prepared at room temperature (Figure 3-A3) or when it is placed in oven at $100{ }^{\circ} \mathrm{C}$ for 60 min (Figure 3C3). Nonetheless, when the solvent is evaporated at $80^{\circ} \mathrm{C}$, a structure formed by spherulites can be observed (Figure 3-B3). On the other hand, the PVDF-HFP@[MIm] $\left[\mathrm{NTf}_{2}\right]$ sample does not show any porosity, regardless of the evaporation temperature of the solvent. Therefore, it is demonstrated that the introduction of the IL on the solution alters the interaction process between the polymer and the solvent in a peculiar way, leading to a variation on the membrane morphology. The 
possibility of tailoring membrane morphology through the variation of the incorporated IL opens new possibilities in the area of membrane production, since the wide range of ILs available with different structures could allow for the design of a huge variety of membrane structures optimized for specific applications.

Regarding the contact angle images shown in Figure 3, it can be appreciated that each sample responds in a different way to the presence of water. This depends on not only the choice of incorporated IL, but also on the synthesis procedure used to fabricate the membranes. Figure 4 shows the contact angle values measured immediately after the contact of the drop with the membrane surface for the

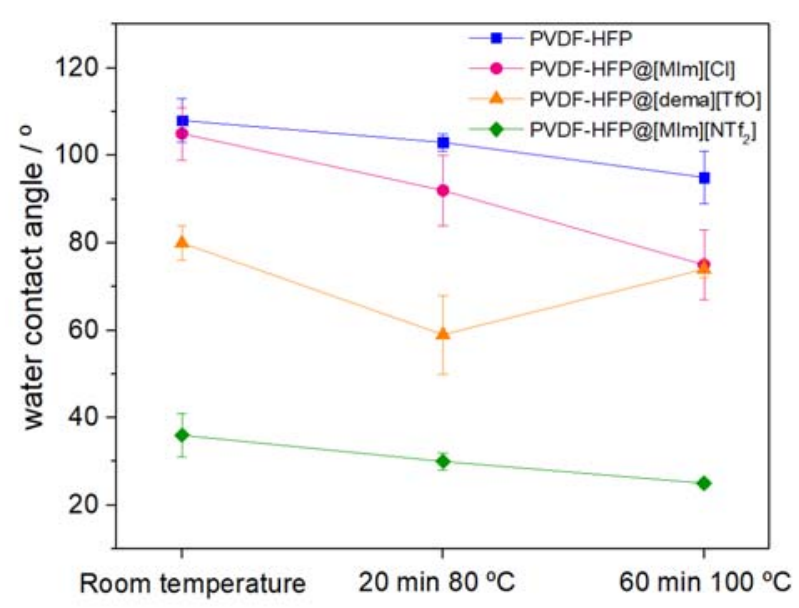
different samples.

Fig. 4 - Contact angle estimated for the samples.

Membranes composed of PVDF-HFP@[MIm][Cl], as well as those of pure PVDF-HFP present higher values of contact angle when compared with the PVDF-HFP@[dema][TfO] and PVDF-HFP@[MIm][NTf $\left.f_{2}\right]$ membranes. Moreover, it is observed that in general the contact angle decreases with increasing the drying temperature of solvent evaporation, as does the porosity of the sample, as observed for PVDF-HFP, PVDF-HFP@[MIm][Cl] and PVDF-HFP@[MIm][NTf $\left.{ }_{2}\right]$. This is because the hydrophobicity of a material is generally modified by the presence of surface textures or roughness [33-34] and the roughness of the prepared samples vary significantly between the different membranes. In contrast, for the composites of PVDF-HFP@[dema][TfO] the smallest contact angle is obtained for the sample prepared at $80{ }^{\circ} \mathrm{C}$. This could be a result of the higher wettability of the samples (analyzed latter), which leads to a huge water absorption rate and a non-correct reading of the contact angle value.

As can be observed the differences between the contact angles and between obtained morphologies are particularly striking in the case of samples dried at $80^{\circ} \mathrm{C}$. Despite being prepared by the same method, the use of different IL results in very different membrane structure. For this reason, the focus of this work is centered around membranes prepared at this temperature.

Furthermore, it is important to also analyze the effect of IL removal on the membrane morphology. As previously described, the samples prepared at $80^{\circ} \mathrm{C}$ were immersed in water for $48 \mathrm{~h}$ in order to remove the IL. The morphology of the membranes before and after the immersion in water is shown in the cross-section images of SEM represented in the Figure 5. 


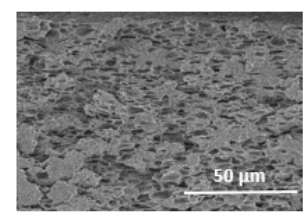

A1

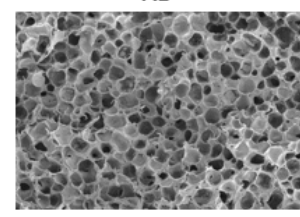

A2

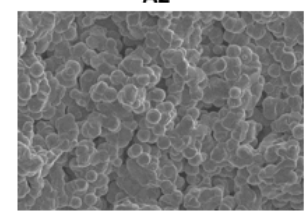

A3

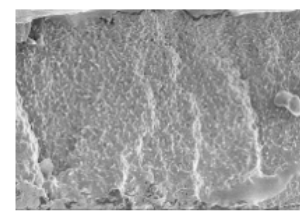

A4

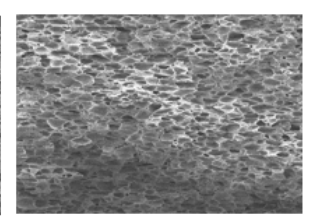

B1

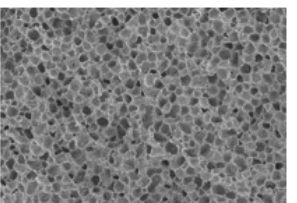

B2

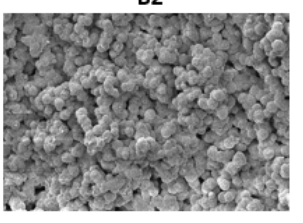

B3

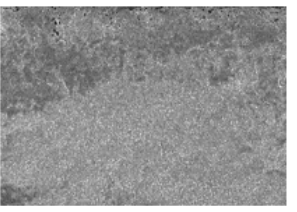

B4

Fig. 5 - Cross-sectional SEM images of the samples dried at $80^{\circ} \mathrm{C}$ before and after immersion in water for $48 \mathrm{~h}(\mathrm{~A}$ and $\mathrm{B}$ respectively). 1, 2, 3 and 4 represent the IL used for the preparation of the membrane (none, [MIm][Cl], [dema][TfO] and $[\mathrm{MIm}]\left[\mathrm{NTf}_{2}\right]$ respectively).

At first glance, the fact that the general morphology is not affected by the removal of IL stands out. In the PVDFHFP@[MIm][Cl] samples, the porous structure is maintained, as well as the spherulitic and compact structures of samples prepared with [dema][TfO] and [MIm] $\left[\mathrm{NTf}_{2}\right]$ ILs, respectively. Nevertheless, a small decrease in the average size of the pores and the spherulites can be observed. It is estimated that the average pore size for the PVDF-HFP@[MIm][Cl] sample before immersion in water is $5.0 \pm 1.3 \mu \mathrm{m}$ while it shrinks to $4.2 \pm 0.8 \mu \mathrm{m}$ after. Regarding the size of the spherulites in the sample of PVDF-HFP@[dema][TfO], their average size before immersion in water is $5.4 \pm 1.1 \mu \mathrm{m}$ and $3.9 \pm 0.8 \mu \mathrm{m}$ after.

Figure 6 shows the images of the contact angle measurements as a function of the time for the PVDF-HFP@IL_80 $80^{\circ}$ samples before and after the immersion in water. In these images, it can be appreciated that the water absorption capacity of the sample is highly dependent from the type of IL incorporated into the composite. The PVDF-HFP@[MIm][Cl] membrane (Figure 6-A1) presents a minimal water absorption capacity after 60s while PVDF-HFP@[dema][TfO] membrane (Figure 6B1) stands out for the significant and rapid capacity of water drop absorption. At a midpoint is the PVDF-HFP@[MIm][NTf $\left.{ }_{2}\right]$ membrane (Figure 6-C1) for which absorption of water over time occurs, while it is not very significant. The removal of IL after the immersion in water (Figures 6-A2, 6-B2 and 6-C2) results in an increase of water contact angle in the three cases. Particularly, the contact angles of PVDF-HFP@[dema][TfO]_ $\mathrm{H}_{2} \mathrm{O}$ and PVDF-HFP@[MIm] $\left[\mathrm{NTf}_{2}\right]_{-} \mathrm{H}_{2} \mathrm{O}\left(134^{\circ}\right.$ and $\left.131^{\circ}\right)$ present a huge increase when compared with the respective composites $\left(92^{\circ}\right.$ and $\left.59^{\circ}\right)$ evidencing the important role played by the IL in the control of the hydrophilicity. Furthermore, these values are even higher than those presented by the directly processed PVDF-HFP membrane $\left(101^{\circ}\right)$ which can be explained by the differences in the roughness of membrane surfaces. 


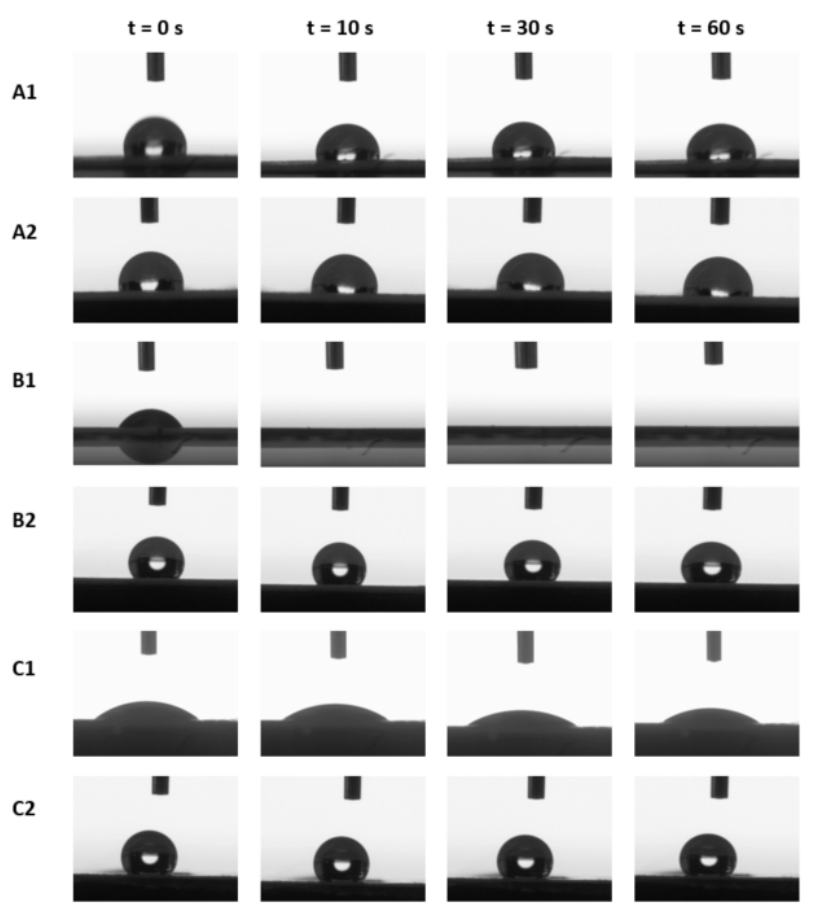

Fig. 6 - Images of the Contact Angle Measurements as a function of time of the different samples dried at $80^{\circ} \mathrm{C}$. A, B and C represent the IL used for the preparation of the membrane ([MIm][Cl], [dema][TfO] and $[\mathrm{MIm}]\left[\mathrm{NTf}_{2}\right]$ respectively) and 1 and 2 represent the membrane before and after the immersion in water.

Finally, we should emphasize that for the membranes where the IL has been removed through water immersion (PVDFHFP@IL_H $\mathrm{H}_{2} \mathrm{O}$ ), the capacity of water absorption decreases with respect to the samples with IL, acquiring a behavior closer to the one of neat PVDF-HFP polymer. In this situation, the variations can be assigned to the membrane morphology. In Figure 7, the quantification of water contact angle of the PVDF-HFP@IL and PVDF-HFP@IL_ $\mathrm{H}_{2} \mathrm{O}$ samples prepared by solvent evaporation at $80^{\circ} \mathrm{C}$ during $20 \mathrm{~min}$ is represented. With the exception of the sample of neat PVDF-HFP, for which difference of water contact angle value before and after the immersion in water is within the measurement error, all the other samples show a higher water contact angle value after the expulsion of the IL from the structure. This phenomenon is particularly significant in the samples of PVDF-HFP@[dema][TfO] and PVDF-HFP@[MIm][NTf 2$]$, which can be explained by the expulsion of hydrophilic ILs from the structure. 


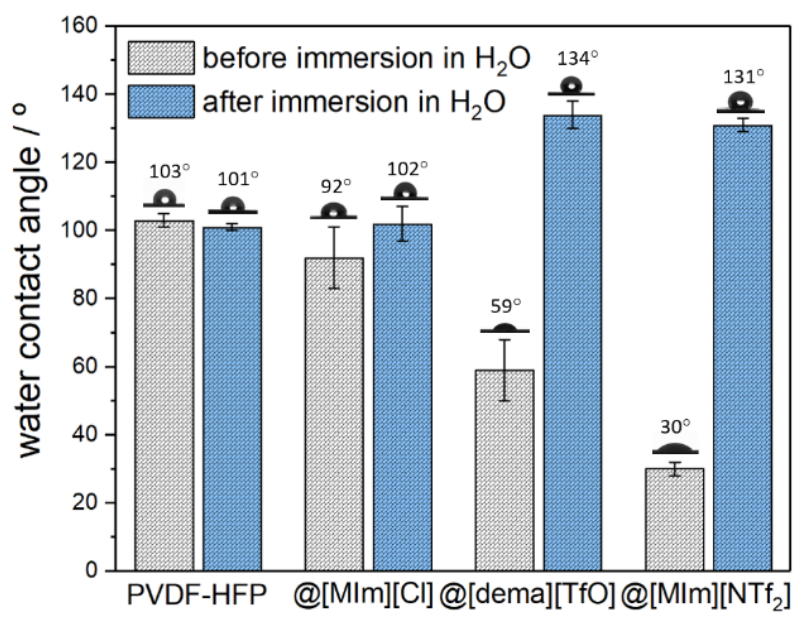

Fig. 7 - Contact angle of the prepared samples before and after immersion in $\mathrm{H}_{2} \mathrm{O}$.

\subsection{Mechanical characterization}

The different morphologies obtained in the samples presented in this study can be also appreciated at the macroscopic scale, where changes in the appearance and mechanical properties of the membranes are observed. The degree of crystallinity and the microstructure of the composite films are the main factor influencing the mechanical properties [35], which are very important for their viability in different applications. To investigate this further, stress-strain curves were measured and are shown in Figure 8-a.
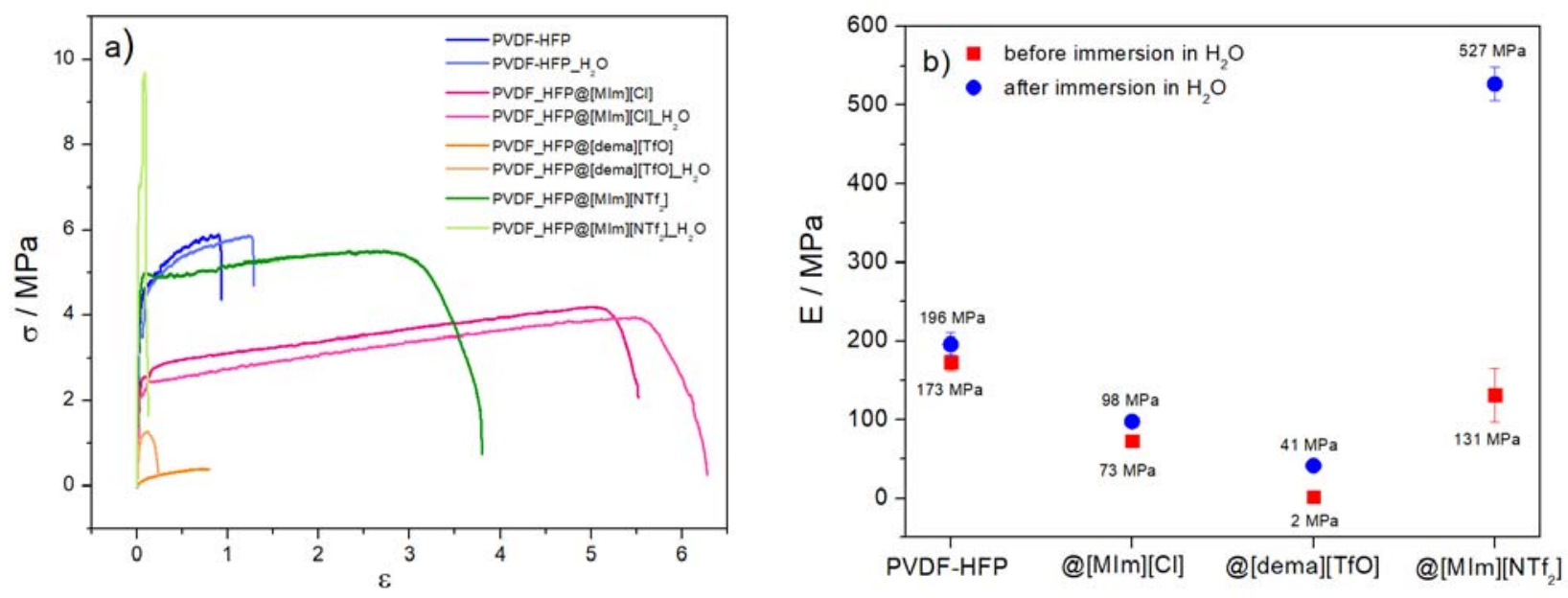

Fig. 8 - (a) Stress-strain curves for the different membranes dried at $80{ }^{\circ} \mathrm{C}$ during 20 min before and after the immersion in water. (b) Results of Young's modulus estimated for the samples whose stress strain curves are shown in Figure 8-a.

As expected, two main characteristic zones are presented in the stress-strain curve. The first zone presents a linear behavior 
that corresponds to the elastic regime and the second zone presents a plastic behavior until the breaking point is reached. From the slope of the linear zone of the curve, the Young's modulus (E) of the sample can be obtained by the application of Hooke's law, $\sigma=E \cdot \varepsilon$, where $\sigma$ and $\varepsilon$ are the stress and the strain, respectively. The results obtained for the Young's modulus of each sample are shown in Figure 8-b. Large variations are observed due to the diversity on the morphology and on the type of the IL present. All the samples prepared with ILs present a higher elasticity than the directly prepared sample of pure PVDF-HFP (173 MPa). The smallest Young's modulus (2 MPa) is obtained for the sample of PVDF-HFP@[dema][TfO] as the spherulitic-based structure of this membrane originates multiple small connection points that can facilitate the stretch process. On the other hand, the highest Young modulus among the IL composite samples is obtained for the membrane of PVDF-HFP@[MIm][NTf $\left.\mathrm{NT}_{2}\right]$ which can be understood as the result of the absence of porous in the structure, which is compact, resulting in a more homogeneous stress distribution across the membrane that makes its deformation more difficult.

Furthermore, important differences are observed in elasticity after the removal of the IL from the membranes. In this case, the variations should be ascribed to the presence or lack of the IL, since the morphology of each membrane is maintained after the removal of the IL. An increase of the Young's modulus is always observed after the immersion of the membranes in water. In the case of PVDF-HFP@[MIm][Cl] and PVDF-HFP@[dema][TfO], the removal of the IL leads to an increase of Young's modulus from 73 to $96 \mathrm{MPa}$ and from 2 to $41 \mathrm{MPa}$, respectively. The most significant variation was observed between the PVDF-HFP@[MIm][NTf $\left.{ }_{2}\right]$ and PVDF-HFP@[MIm][NTf $\left.{ }_{2}\right] \mathrm{H}_{2} \mathrm{O}$ samples. The removal of [MIm][NTf $\left.\mathrm{M}_{2}\right]$ from these membranes results in an increase of 300\% in the Young's modulus. The plasticizer effect of ILs discussed in other studies in the literature [36] is consistent with these results. However, our results show that the membrane morphology also plays an important role in determining elastic properties of these materials, as an increase in the Young's modulus is still obtained compared to the pure PVDF-HFP samples even after the ILs have been removed from the composite membranes, as can be observed for the PVDF-HFP@[MIm] $\left[\mathrm{NTf}_{2}\right]_{-} \mathrm{H}_{2} \mathrm{O}$ sample, which shows a 63\% increase when compared with to pure PVDF-HFP. Both are free of IL but vary in morphology: the absence of pores in the PVDF-HFP@[MIm][NTf $\left.{ }_{2}\right] \mathrm{H}_{2} \mathrm{O}$ membrane structure is a determinant factor.

\subsection{Thermal characterization}

All the previous results indicate that, despite the different morphologies, part of neat PVDF-HFP's characteristics can be somewhat recovered after the removal of the IL. To further understand the thermal behavior of the samples as well as the PVDF-HFP and IL interactions, thermogravimetric curves of neat PVDF-HFP and of PVDF-HFP@IL_80 ${ }^{\circ} \mathrm{C}$ composites were recorded (Figure 9-a). The analysis of the results shows the effect of the different ILs on the degradation temperatures of the membranes. The same analysis was performed in the samples of PVDF-HFP@IL_ $\mathrm{H}_{2} \mathrm{O} \_80^{\circ} \mathrm{C}$, which IL was removed (Figure 9-b). 

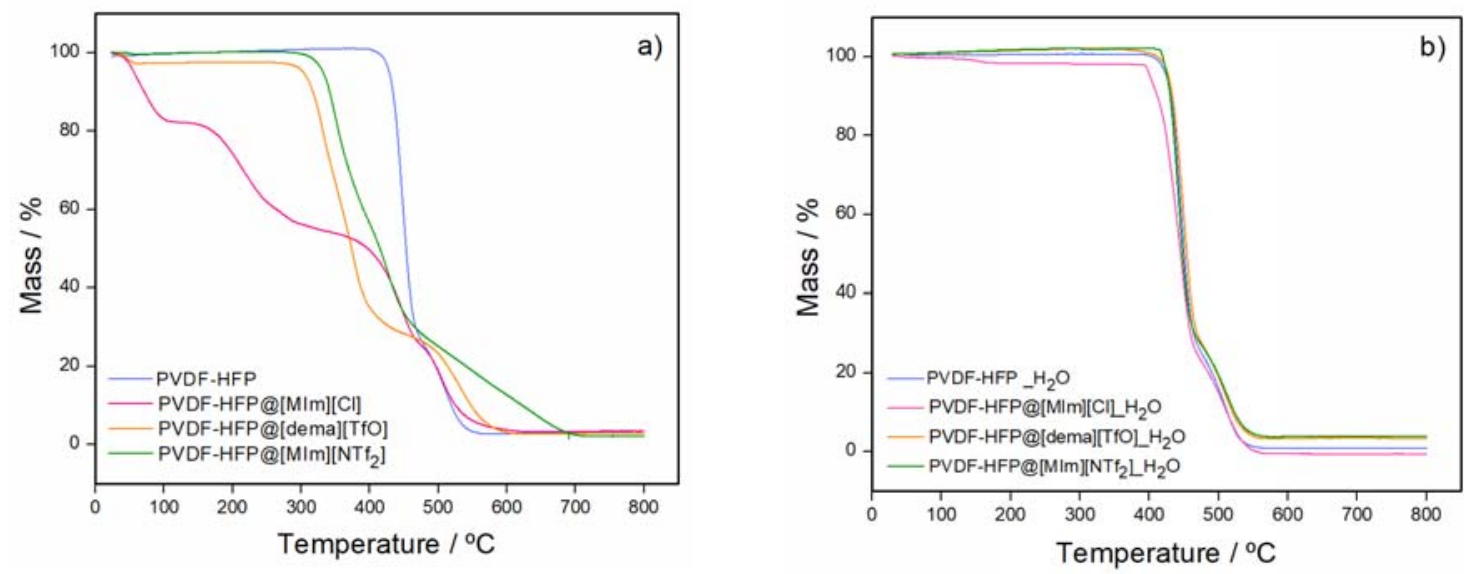

Fig. 9 - Thermogravimetric curves for the membranes dried at $80{ }^{\circ} \mathrm{C}$, (a) before immersion in water (b) after immersion in water.

TGA measurements demonstrate that the pure PVDF-HFP membrane is thermally stable until temperatures close to 450 ${ }^{\circ} \mathrm{C}$. At this temperature, the carbon-hydrogen bonds of the polymer chain break, and the formation and loss of hydrogen fluoride along the polymer chain occurs [37]. Other previous weight losses processes occur on PVDF-HFP@IL composites which are related with the ionic liquids degradation.

PVDF-HFP@[dema][TfO] and PVDF-HFP@[MIm][NTf $\left.{ }_{2}\right]$ composites present the more significant weight loss at an onset temperature above $300^{\circ} \mathrm{C}$ which is due to the degradation of the IL [38]. The PVDF-HFP@[dema][TfO] sample presents an additional small initial weight loss $(3 \% \mathrm{wt})$ at lower temperatures, around $100^{\circ} \mathrm{C}$, most likely corresponding to the loss of the water present in this IL structure. However, the most significant differences are observed for the PVDF-HFP@[MIm][Cl] composite. This sample presents an initial mass loss of about $18 \%$ wt around $100{ }^{\circ} \mathrm{C}$. Such as in the previous case, this corresponds to the loss of the water present in the hydrophilic IL. Contrary to other composites, the one with [MIm][Cl] presents another mass loss step of about $25 \%$ wt below $200{ }^{\circ} \mathrm{C}$, followed by the last degradation process at temperatures around $450{ }^{\circ} \mathrm{C}$. This anticipation of degradation process is typical in samples containing $\mathrm{Cl}$ ions, which present less thermal stability than the other two ILs [39].

The results of PVDF-HFP@IL_H $\mathrm{H}_{2} \mathrm{O} \_80^{\circ} \mathrm{C}$ samples, where the ILs have been removed after the water immersion process, are represented in Figure 8-b. As expected, a change is noted in the thermal degradation temperature when compared to the respective composites. The absence of IL transforms these membranes into neat PVDF-HFP membranes, leading to a considerable increase in the degradation temperature values close to the ones of the originally prepared pure PVDF-HFP membrane.

\subsection{Fourier Transform Infrared Spectroscopy}

The combination of different morphologic structures with the piezoelectric properties of PVDF-HFP can be an added value to widen the application possibilities of this polymer. However, inducing the piezoelectric crystal phase ( $\beta$ or $\gamma)$ in PVDF-HFP can be a tricky process, especially if non-porous structures are desired [9]. Recent studies describe the capacity of ILs to induce a piezoelectric phase in PVDF even under conditions that usually result in the non-piezoelectric $\alpha$-phase 
[40]. In order to identify the crystalline phase of the composite membranes presented in this work, FTIR-ATR measurements were performed and are presented in Figure 10.
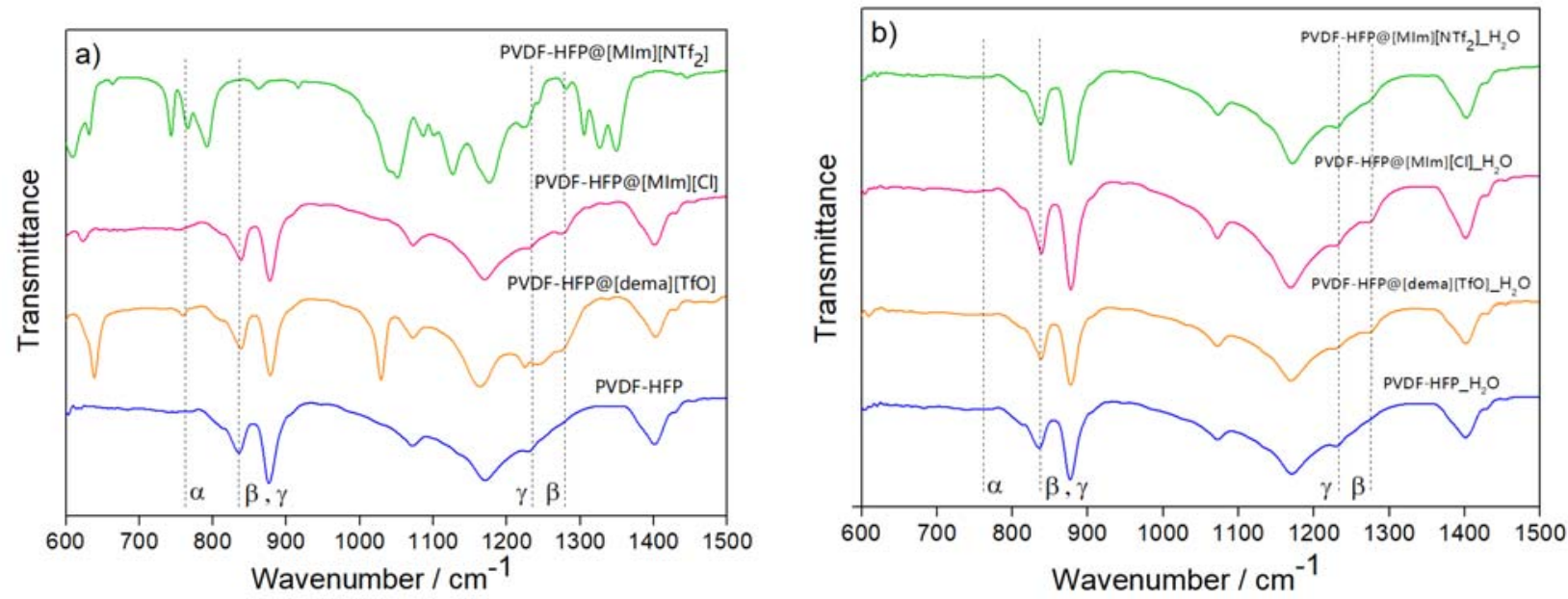

Fig. 10 - FTIR-ATR spectra of the different membranes dried at $80^{\circ} \mathrm{C}$, (a) before immersion in water (b) after immersion in water.

As shown in Figure 10-a, it can be observed that the characteristic bands of non-piezoelectric $\alpha$-phase $(766,976,1149$ and $1383 \mathrm{~cm}^{-1}$ ) are not present in the samples of neat PVDF-HFP and PVDF-HFP@[MIm][Cl]. Nevertheless PVDFHFP@[dema][TfO] and PVDF-HFP@[MIm] $\left[\mathrm{NTf}_{2}\right]$ composites present a small band around $766 \mathrm{~cm}^{-1}$. This band, along with those detected at 976,1149 or $1383 \mathrm{~cm}^{-1}$, is a common characteristic of IL vibrations. On the other hand, the FTIR-ATR spectrum of all the composites, with exception to the PVDF-HFP@[MIm][NTf 2 , presents an intense band at $840 \mathrm{~cm}^{-1}$, typical of the piezoelectric $\beta$ and $\gamma$-phases. In parallel, low intense bands at 1233 and $1279 \mathrm{~cm}^{-1}$, characteristic of $\gamma$ and $\beta$-phases, respectively, can also be observed, diluted among other non-specific bands [9]. The presence of no characteristic bands of PVDF-HFP is a phenomenon that stands out in the case of PVDF-HFP@[MIm] $\left[\mathrm{NTf}_{2}\right]$ sample. These bands can be associated with the presence of different ILs or with an incomplete crystallization process of the polymer when in the presence of the IL.

Significant changes in the FTIR-ATR spectra of the composite membranes are observed after removing the ILs through the water immersion process. The FTIR-ATR spectra of PVDF-HFP@IL_ $\mathrm{H}_{2} \mathrm{O}$ samples (Figure 10-b) are very similar to the directly produced pure membrane of PVDF-HFP. Only the characteristic bands of the polymer are present, confirming the removal of the IL from the membrane structure. Moreover, we observe that the characteristic bands of piezoelectric $\gamma$ and $\beta$ phases become more intensive and clear. This is observed in all the immersed samples, even in PVDFHFP@[MIm] $\left.\mathrm{NTf}_{2}\right]_{-} \mathrm{H}_{2} \mathrm{O} \_80^{\circ} \mathrm{C}$, which did not previously present any characteristic band of the piezoelectric phases. This suggests that the process of polymer crystallization into these phases is only completed after the removal of [MIm] [NTf $\left.\mathrm{N}_{2}\right]$ from the matrix. Additionally, the presence of the 1234 and $1279 \mathrm{~cm}^{-1}$ bands points to a mix of the $\gamma$ and $\beta$-phase in the polymer structure. The coexistence of these two phases prevents their relative quantification, since actual quantification methods only consider the coexistence of $\alpha$ and $\beta$-phases or $\alpha$ and $\gamma$-phases [9]. Either way, the absence of $\alpha$-phase's characteristic bands indicates a complete crystallization of PVDF-HFP@IL_ $\mathrm{H}_{2} \mathrm{O} \_80^{\circ} \mathrm{C}$ into piezoelectric phases, underlining 
how using ILs during fabrication can achieve specific morphologies and crystal properties and open the possibility of tailoring these materials to a wide variety of applications.

\section{Conclusions}

PVDF-HFP membranes with different ILs ([MIm][Cl], [dema][TfO] and $\left.[\mathrm{MIm}]\left[\mathrm{NTf}_{2}\right]\right)$ were produced by solvent casting at different temperatures. ILs are shown to serve as properties controllers on the production of PVDF-HFP membranes. Aspects such as morphology, water absorption capacity and mechanical properties have been shown to depend not only from the production methodology employed, but also from the type of IL incorporated in the membranes. Porous, spherulitic or compact polymer structures were obtained by solvent evaporation at $80^{\circ} \mathrm{C}$ during 20 min, using $[\mathrm{MIm}][\mathrm{Cl}]$, [dema][TfO] and $[\mathrm{MIm}]\left[\mathrm{NTf}_{2}\right]$ IL respectively. All the prepared composites show a lower water contact angle when compared to neat PVDF-HFP. The PVDF-HFP@[MIm] $\left[\mathrm{NTf}_{2}\right]$ composite presents the lowest contact angle, followed by PVDFHFP@[dema][TfO] and PVDF-HFP@[MIm][NTf 2$]$. Particularly, PVDF-HFP@[dema][TfO] stands out for its fast water absorption capacity as well as for the very low of Young's modulus ( $2 \mathrm{MPa}$ ) related to its spherulitic morphology. Finally, we demonstrate that the introduction of the composites in water leads to the removal of the IL present in the polymer matrix, which conserves the morphology of the produced membrane and recovers most of the typical characteristics of the neat PVDF-HFP (hydrophobicity, thermal stability, piezoelectric crystallinity, among others), and can also be used to induce piezoelectric phases in the material. The possibility of tailoring PVDF-HFP membranes morphology through the variation in the choice of the introduced IL is of particular interest and opens new possibilities in the area of membranes production, particularly the extensive range of ILs commercially available means that a wide variety of membrane structures could be obtained through this approach. The appropriate choice of IL and production method will lead to the achievement of desired properties and allow the application of polymer membranes in fields where their morphological properties need to be optimized.

\section{Acknowledgements}

This work was financially supported by the "Ministerio de Economía, Industria y Competitividad" (MAT201676739R(AEI/FEDER, UE)) and by the "Gobierno Vasco" (Basque University Research System Group, IT-630-13). The technical and human support provided by SGiker (UPV/EHU) is gratefully acknowledged. P.GSaiz thanks the BCMaterials center for the formation scholarship and to the Dr. J.L. Vilas for providing the equipment for contact angle measurements. S. Eizagirre thanks the European Union support received under Erasmus + Programme. A.C.Lopes thanks to MSCA-IF-2015 (Marie Skłodowska Curie Actions) of the European Union's Horizon 2020 Programme for the received funds under grant agreement $\mathrm{n}^{\circ}[701852]$.

\section{REFERENCES}


[1] W. Zhou, A. Bahi, Y. Li, H. Yang, and F. Ko, "Ultra-filtration membranes based on electrospun poly(vinylidene fluoride) (PVDF) fibrous composite membrane scaffolds," RSC Adv., vol. 3, no. 29, pp. 11614-11620, 2013.

[2] V. T. Rathod, D. R. Mahapatra, A. Jain, and A. Gayathri, "Characterization of a large-area PVDF thin film for electromechanical and ultrasonic sensing applications," Sensors Actuators, A Phys., vol. 163, no. 1, pp. 164-171, 2010.

[3] C. Ribeiro, V. Sencadas, D. M. Correia, and S. Lanceros-Mendez, "Piezoelectric polymers as biomaterials for tissue engineering applications," Colloids Surfaces B Biointerfaces, vol. 136, pp. 46-55, 2015.

[4] Z. Jin, X. Wang, and X. Cui, "Synthesis and morphological investigation of ordered SBA-15-type mesoporous silica with an amphiphilic triblock copolymer template under various conditions," Colloids Surfaces A Physicochem. Eng. Asp., vol. 316, no. 1-3, pp. 27-36, 2008.

[5] D. Wang, "A novel method for controlling the surface morphology of polymeric membranes," J. Memb. Sci., vol. 169, no. 1, pp. 39-51, 2000.

[6] Y. S. Yang Yang Jie Liu and Tzung-Fang Guo, "The control of morphology and the morphological dependence of device electrical and optical properties in polymer electronics," Electronic and Optical Properties of Conjugated Molecular Systems in Condensed Phases, vol. 661, no. 2, pp. 307-354 2003.

[7] Y. Shi, J. Liu, and Y. Yang, "Device performance and polymer morphology in polymer light emitting diodes: The control of thin film morphology and device quantum efficiency,” J. Appl. Phys., vol. 87, no. 9, pp. 4254-4263, 2000.

[8] C. G. Ferreira, V. F. Cardoso, A. C. Lopes, G. Botelho, and S. Lanceros-Méndez, "Tailoring microstructure and physical properties of poly(vinylidene fluoride-hexafluoropropylene) porous films," J. Mater. Sci., vol. 50, no. 14, pp. 5047-5058, 2015.

[9] P. Martins, A. C. Lopes, and S. Lanceros-Mendez, "Electroactive phases of poly(vinylidene fluoride): Determination, processing and applications," Prog. Polym. Sci., vol. 39, no. 4, pp. 683-706, 2014.

[10] A. H. Rajabi, M. Jaffe, and T. L. Arinzeh, "Piezoelectric materials for tissue regeneration: A review," Acta Biomater., vol. 24, pp. 12-23, 2015.

[11] L. Shi, R. Wang, Y. Cao, C. Feng, D. T. Liang, and J. H. Tay, "Fabrication of poly(vinylidene fluoride-cohexafluropropylene) (PVDF-HFP) asymmetric microporous hollow fiber membranes," J. Memb. Sci., vol. 305, no. $1-2$, pp. 215-225, 2007.

[12] A. Du Pasquier, P. C. Warren, D. Culver, A. S. Gozdz, G. G. Amatucci, and J. M. Tarascon, "Plastic PVDF-HFP electrolyte laminates prepared by a phase-inversion process," Solid State Ionics, vol. 135, no. 1-4, pp. 249-257, 2000 .

[13] D. Hou, D. Lin, C. Ding, D. Wang, and J. Wang, "Fabrication and characterization of electrospun superhydrophobic PVDF-HFP/SiNPs hybrid membrane for membrane distillation,” Sep. Purif. Technol., vol. 189, no. July, pp. 82-89, 2017.

[14] G. A. Buxton and N. Clarke, "Ordering polymer blend morphologies via solvent evaporation," Europhys. Lett., vol. 78 , no. 5, p. 56006, 2007.

[15] M. Gu, J. Zhang, X. Wang, H. Tao, and L. Ge, "Formation of poly(vinylidene fluoride) (PVDF) membranes via thermally induced phase separation," Desalination, vol. 192, no. 1-3, pp. 160-167, 2006.

[16] X. Li, Y. Wang, X. Lu, and C. Xiao, "Morphology changes of polyvinylidene fluoride membrane under different phase separation mechanisms," J. Memb. Sci., vol. 320, no. 1-2, pp. 477-482, 2008.

[17] P. Y. Zhang, H. Yang, Z. L. Xu, Y. M. Wei, J. L. Guo, and D. G. Chen, "Characterization and preparation of 
poly(vinylidene fluoride) (PVDF) microporous membranes with interconnected bicontinuous structures via nonsolvent induced phase separation (NIPS)," J. Polym. Res., vol. 20, no. 2, 2013.

[18] M. J. Koh, H. Y. Hwang, D. J. Kim, H. J. Kim, Y. T. Hong, and S. Y. Nam, "Preparation and Characterization of Porous PVdF-HFP/clay Nanocomposite Membranes,” J. Mater. Sci. Technol., vol. 26, no. 7, pp. 633-638, 2010.

[19] K. M. Kim, N.-G. Park, K. S. Ryu, and S. H. Chang, "Characterization of poly (vinylidenefluoride-cohexafluoropropylene)-based polymer electrolyte filled with $\mathrm{TiO}_{2}$ nanoparticles,” Polymer, vol. 43, no. 14, pp. 3951$3957,2002$.

[20] J. C. Dias, D. C. Correia, A. C. Lopes, S. Ribeiro, C Ribeiro, V. Sencadas, G. Botelho, J. M. S. S. Esperança, J. M. Laza, J. L. Vilas, L. M. Leon, ans S. Lanceros-Mendez, "Development of poly(vinylidene fluoride)/ionic liquid electrospun fibers for tissue engineering applications," J. Mater. Sci., vol. 51, no. 9, pp. 4442-4450, 2016.

[21] T. Welton, "Room-Temperature Ionic Liquids. Solvents for Synthesis and Catalysis,” Chem. Rev., vol. 99, no. 8, pp. 2071-2084, 1999.

[22] M. Watanabe, "Design and Materialization of Ionic Liquids Based on an Understanding of Their Fundamental Properties," Electrochemistry, vol. 84, no. 9, pp. 642-653, 2016.

[23] J. E. Bara, S. Lessmann, C. J. Gabriel, E. S. Hatakeyama, R. D. Noble, and D. L. Gin, "Synthesis and performance of polymerizable room-temperature ionic liquids as gas separation membranes," Ind. Eng. Chem. Res., vol. 46, no. 16, pp. 5397-5404, 2007.

[24] K. Fujita, D. R. MacFarlane, and M. Forsyth, "Protein solubilising and stabilising ionic liquids," Chem. Commun., vol. 70, no. 38, pp. 4804-4806, 2005.

[25] J. Zhang, J. Wang, S. Zhou, K. Duan, B. Feng, J. Weng, and P. Wu, "Ionic liquid-controlled synthesis of ZnO microspheres," J. Mater. Chem., vol. 20, no. 43, p. 9798, 2010.

[26] R. Bussamara, W. W. Melo, J. D. Scholten, P. Migowski, G. Marin, M.J. M. Zapata, G. Machado, S. R. Teixeira, M. A. Novak, and J Dupont “Controlled synthesis of $\mathrm{Mn}_{3} \mathrm{O}_{4}$ nanoparticles in ionic liquids,” Dalt. Trans., vol. 42, no. 40, p. 14473, 2013.

[27] M. Antonietti, D. Kuang, B. Smarsly, and Y. Zhou, "Ionic liquids for the convenient synthesis of functional nanoparticles and other inorganic nanostructures," Angew. Chemie - Int. Ed., vol. 43, no. 38, pp. 4988-4992, 2004.

[28] D. S. Lakshmi, T. Cundari, E. Furia, A. Tagarelli, G. Fiorani, M. Carraro, and A. Figoli, "Preparation of polymeric membranes and microcapsules using an ionic liquid as morphology control additive," Macromol. Symp., vol. 357, no. 1, pp. 159-167, 2015.

[29] B. Ma, J. Yang, Q. Sun, W. Jakpa, X. Hou, and Y. Yang, "Influence of cellulose/[Bmim]Cl solution on the properties of fabricated NIPS PVDF membranes,” J. Mater. Sci., vol. 52, no. 16, pp. 9946-9957, 2017.

[30] C. Xing, M. Zhao, L. Zhao, J. You, X. Cao, and Y. Li, “Ionic liquid modified poly(vinylidene fluoride): crystalline structures, miscibility, and physical properties," Polym. Chem., vol. 4, no. 24, pp. 5726-5734, 2013.

[31] J. F. Brennecke and E. J. Maginn, “Ionic liquids: Innovative fluids for chemical processing,” AIChE J., vol. 47, no. 11, pp. 2384-2389, 2001.

[32] R. E. Sousa, J. C. C. Ferreira, C. M. Costa, A. V. Machado, M. M. Silva, and S. Lanceros-Mendez, "Tailoring poly(vinylidene fluoride-co-chlorotrifluoroethylene) microstructure and physicochemical properties by exploring its binary phase diagram with dimethylformamide," J. Polym. Sci. Part B Polym. Phys., vol. 53, no. 11, pp. 761-773, 2015. 
[33] J. T. Simpson, S. R. Hunter, and T. Aytug, "Superhydrophobic materials and coatings: a review," Reports Prog. Phys., vol. 78, no. 8, p. 86501, 2015.

[34] V. F. Cardoso, A. R. Machado, V. C. Pinto, P. J. Sousa, G. Botelho, G. Minas, and S. Lanceros-Méndez, "From superhydrophobic- to superhydrophilic-patterned poly(vinylidene fluoride- $c o$-chlorotrifluoroethylene) architectures as a novel platform for biotechnological applications," J. Polym. Sci. Part B Polym. Phys., pp. 1-9, 2016.

[35] V. F. Cardoso, G. Botelho, and S. Lanceros-Méndez, "Nonsolvent induced phase separation preparation of poly(vinylidene fluoride-co-chlorotrifluoroethylene) membranes with tailored morphology, piezoelectric phase content and mechanical properties," Mater. Des., vol. 88, pp. 390-397, 2015.

[36] K. Park, J. U. Ha, and M. Xanthos, "Ionic liquids as plasticizers/lubricants for polylactic acid," Polymer Engineering Science, vol. 50, pp. 1105-1110, 2010.

[37] G. Botelho, S. Lanceros-Mendez, A. M. Gonçalves, V. Sencadas, and J. G. Rocha, "Relationship between processing conditions, defects and thermal degradation of poly(vinylidene fluoride) in the $\beta$-phase," J. Non. Cryst. Solids, vol. 354, no. 1, pp. 72-78, 2008.

[38] H. Nakamoto and M. Watanabe, "Brønsted acid-base ionic liquids for fuel cell electrolytes," Chem. Commun., no. 24, pp. 2539-2541, 2007.

[39] H.-J. Liaw, C.-C. Chen, Y.-C. Chen, J.-R. Chen, S.-K. Huang, and S.-N. Liu, "Relationship between flash point of ionic liquids and their thermal decomposition," Green Chem., vol. 14, no. 7, pp. 2001-2008, 2012.

[40] A. C. Lopes, J. Gutiérrez, and J. M. Barandiarán, "Direct fabrication of a 3D-shape film of polyvinylidene fluoride (PVDF) in the piezoelectric $\beta$-phase for sensor and actuator applications," Eur. Polym. J., no. 99, pp. 111-116, 2018.

[41] M. J. Park, I. Choi, J. Hong, and O. Kim, "Polymer electrolytes integrated with ionic liquids for future electrochemical devices,” J. Appl. Polym. Sci., vol. 129, no. 5, pp. 2363-2376, 2013. 